\title{
A Generalized Normal Form and its Application to Sliding Mode Control
}

\author{
Håkan Fortell \\ Department of Electrical Engineering \\ Linköping University \\ S-581 83 Linköping, Sweden \\ Phone: +4613284028 \\ E-mail: hafor@isy.liu.se
}

April 4, 1995

\begin{abstract}
In this report it is shown how a normal form, corresponding to that of affine state space systems, can be calculated for generalized state space descriptions. The calculations are performed by using Gröbner bases. This normal form is then used in the context of sliding mode control. The result generalizes the previous results on sliding mode control for generalized state space representations.
\end{abstract}

Keywords: Normal Forms, Sliding Mode Control, Gröbner Bases

\section{Introduction}

In [6] it is shown how a normal form can be calculated for generalized state space descriptions [4]. A thing to investigate is then if this normal form can be used in similar ways as the normal form of affine state space systems [7]. One such application is in the context of sliding mode control, see ,e.g., [10]. Constructing sliding mode controllers for generalized state space representations has already been considered in [12] where the generalized controller canonical form as described in [2] is used. The problem with this approach is that one is restricted to zeroing a quite restricted class of elements. The order of the differential equation that must be solved to get the sliding control law is also of maximal order of the input. The purpose of this report is to try to find sliding mode control laws for a larger class of manifolds and to see if one can find differential equation for the input which is of an order lower than the maximal one. The paper is organized as follows. The next section contains some of the most basic concepts from commutative and differential algebra. Section 3 then shows how a generalized normal form can be computed and in section 4 this is applied to sliding mode control. Finally in section 5 we give some concluding remarks. 


\section{Basic Algebraic Concepts}

In this section we present some of the most basic concepts from commutative and differential algebra that will be used in later sections. The purpose is basically to display the notation.

\subsection{Commutative Algebra}

The theory in this section is collected from [1] and [5] where all proofs that are left out and also further references to commutative algebra can be found. We begin by making a few necessary definitions.

\section{Definition 2.1}

A commutative ring consists of a set $R$ and two binary operations "." and "+" defined on $R$ and such that:

1. $(a+b)+c=a+(b+c)$ and $(a \cdot b) \cdot c=a \cdot(b \cdot c)$ for all $a, b, c \in R$.

2. $a+b=b+a$ and $a \cdot b=b \cdot a$ for all $a, b \in R$.

3. $a \cdot(b+c)=a \cdot b+a \cdot c$ for all $a, b, c \in R$.

4. There are $0,1 \in R$ such that $a+0=a \cdot 1=a$ for all $a \in R$.

5. Given $a \in R$ there is $b \in R$ such that $a+b=0$.

We also need the following.

\section{Definition 2.2}

$A$ field $k$ is a commutative ring such that given $a \in k$ there is $c \in k$ such that $a \cdot c=1$.

The commutative ring that we will mostly work with here is $k\left[x_{1}, \ldots, x_{n}\right]$ which denotes the set of all polynomials in the variables $x_{1}, \ldots, x_{n}$ with coefficients taken from the field $k$. Usually we will assume that $k$ is the real numbers.

\section{Definition 2.3}

A subset $I$ of a commutative ring $R$ is an ideal if it satisfies

1. $0 \in I$.

2. If $a, b \in I$ then $a+b \in I$.

3. If $a \in I$ and $b \in R$ then $b \cdot a \in I$.

If we now set $f_{1}, \ldots, f_{\bar{n}}$ to be polynomials in a ring $k\left[x_{1}, \ldots, x_{n}\right]$ and introduce the notation

$$
\left\langle f_{1}, \ldots, f_{\bar{n}}\right\rangle=\left\{\sum_{i=1}^{\bar{n}} p_{i} f_{i}: p_{i} \in k\left[x_{1}, \ldots, x_{n}\right], i=1, \ldots, \bar{n}\right\}
$$

we can note that $\left\langle f_{1}, \ldots, f_{\bar{n}}\right\rangle$ is an ideal and it is called the ideal generated by $f_{1}, \ldots, f_{\bar{n}}$.

A property that will be needed in the next section is to have some generalization of linear dependence of polynomials. To find such a generalization we first need some more definitions. 


\section{Definition 2.4}

If the field $k$ is a subset of the field $K$ then $K$ is said to be an extension field of $k$. This is denoted by $K / k$.

\section{Definition 2.5}

Let $K$ be an extension field of $k$ and suppose that $\alpha \subset K$. The smallest field containing $k \cup \alpha$ is then called the field obtained by adjoining $\alpha$ to $k$ and is denoted $k(\alpha)$. Furthermore, $\alpha$ is said to be algebraic over $k$ if there exists a nonzero polynomial $f \in k[x]$ such that $f(\alpha)=0$. If $\alpha$ is not algebraic it is said to be transcendental over $k$.

A definition can also be made in a similar manner for several elements in an extension field.

\section{Definition 2.6}

Let $f_{1}, \ldots, f_{m}$ be elements in an extension field $K$ of $k$. The $f_{i}$ are said to be algebraically dependent over $k$ if there exists a nonzero $P \in k\left[x_{1}, \ldots, x_{m}\right]$ such that $P\left(f_{1}, \ldots, f_{m}\right)=0$. Otherwise the $f_{i}$ are said to be algebraically independent.

Since algebraic dependence can be viewed as a generalization of linear dependence in linear algebra we can go on to define a basis for these extension fields.

\section{Definition 2.7}

Let $K$ be an extension field of $k$ and let $U$ be a subset of $K$ such that all elements of $U$ are algebraically independent. The maximum number of elements that such a $U$ can contain is called the transcendence degree of $K / k$ and is denoted by trdeg $K / k$. The elements of such a $U$ is called the transcendence basis for $K / k$.

An important consequence of this definition is then the theorem below.

\section{Theorem 2.1}

If $k$ is a field and the variables $x_{i}, i=1, \ldots, n$ are algebraically independent over $k$ then $\operatorname{trdeg} k\left(x_{1}, \ldots, x_{n}\right) / k=n$.

\section{Proof.}

We only have to note that the $x_{i}, i=1, \ldots, n$ is a transcendence basis for $k\left(x_{1}, \ldots, x_{n}\right) / k$.

The following corollary will also be useful.

Corollary 2.1 The elements $y_{1}, \ldots, y_{N} \in k\left(x_{1}, \ldots, x_{n}\right)$ are algebraically dependent if $N>n$.

\section{Proof.}

Otherwise $y_{1}, \ldots, y_{N}$ would be a transcendence basis for $k\left(x_{1}, \ldots, x_{n}\right) / k$.

Since algebraic dependence will be an important concept later on we give a theorem related to this subject.

Theorem 2.2

Suppose $k$ is a field of characteristic zero and that $f_{1}, \ldots, f_{N} \in k\left(x_{1}, \ldots, x_{n}\right)$. Then if 
- $N>n, f_{1}, \ldots, f_{N}$ are algebraically dependent.

- $N=n, f_{1}, \ldots, f_{N}$ are algebraically dependent iff the Jacobian matrix

$$
J(f)=\left(\frac{\partial f_{i}}{\partial x_{j}}\right)_{i j}
$$

is identically singular.

- $N<n, f_{1}, \ldots, f_{N}$ are algebraically independent iff the Jacobian matrix (2) has rank $N$.

\subsection{Gröbner bases}

The reason why we introduce the concept of Gröbner bases is that we wish to have some way of eliminating variables from a set of polynomial equation. To be able to choose which variables to eliminate we must introduce an ordering of variables.

\section{Definition 2.8}

Let $x^{\alpha} \in k\left[x_{1}, \ldots, x_{n}\right]$ with $\alpha=\left\{\alpha_{1}, \ldots, \alpha_{n}\right\} \in \mathbb{N}^{n}$ denote $x_{1}^{\alpha_{1}} \cdots x_{n}^{\alpha_{n}}$. A term ordering $<$ is an ordering on $\mathbb{N}^{n}$ such that for all $\alpha, \beta, \gamma \in \mathbb{N}^{n}$

$$
0<\alpha \text { and } \alpha<\beta \Rightarrow \alpha+\gamma<\beta+\gamma
$$

There are of course several ways of defining these term orderings but we will only consider one of them.

\section{Definition 2.9}

The lexicographic term ordering is defined by

$$
\alpha<\beta \Longleftrightarrow \exists j: \alpha_{j}<\beta_{j}, \forall i<j: \alpha_{i}<\beta_{i}
$$

With a term ordering will now the different terms in a polynomial be ordered. In particular will one of them be ordered first.

\section{Definition 2.10}

If $f=\sum c_{\gamma} x^{\gamma} \in k\left[x_{1}, \ldots, x_{n}\right]$ then the degree of $f$ is defined as

$$
\operatorname{deg} f=\max \left\{\gamma: c_{\gamma} \neq 0\right\}
$$

The leading term of $f$ is

$$
L T f=c_{\operatorname{deg} f} x^{\operatorname{deg} f}
$$

and correspondingly the leading monomial of $f$ is $L M f=x^{\operatorname{deg} f}$ and the leading coefficient is $L C f=c_{d e g} f$.

This definition can now be extended to cover ideals.

Definition 2.11

Let $\mathcal{I}$ be an ideal in $k\left[x_{1}, \ldots, x_{n}\right]$. Then

$$
\begin{aligned}
L M \mathcal{I} & =\langle\operatorname{LM} f: f \in \mathcal{I}\rangle \\
\operatorname{deg} \mathcal{I} & =\{\operatorname{deg} f: \in \mathcal{I}\}
\end{aligned}
$$


Using this last definition we can now go on to define Gröbner bases.

\section{Definition 2.12}

$A$ set $G \subseteq \mathcal{I}$ for an ideal $\mathcal{I}$ is a Gröbner base with respect to a given term ordering iff $L M \mathcal{I}=\langle L M G\rangle$.

At a first glance it not at all clear how the concept of Gröbner bases can be useful but it can be shown to have several nice properties. One of these properties is that

$$
\langle G\rangle=\mathcal{I}
$$

which says that the set of solutions to the generating set of $\mathcal{I}$ is the same for the equations described by the Gröbner base. To be able to describe the algorithm we need to introduce a few more concepts.

\section{Definition 2.13}

Let $f, g \in k\left[x_{1}, \ldots, x_{n}\right] . f$ is said to be reduced w.r.t. $g$ if there is no term in $f$ that is divisible by the leading term of $g$. A subset of $k\left[x_{1}, \ldots, x_{n}\right]$ in which all elements are reduced w.r.t. each other is said to be auto-reduced

If some polynomials are not reduced we can make them reduced.

Theorem 2.3

Let $F=\left\{f_{1}, \ldots, f_{m}\right\} \subset k\left[x_{1}, \ldots, x_{n}\right]$ and $p \in k\left[x_{1}, \ldots, x_{n}\right]$. Then it is possible to find polynomials $g_{1}, \ldots, g_{m}, r \in k\left[x_{1}, \ldots, x_{n}\right]$ such that

$$
r=p-\sum_{i=1}^{m} g_{i} f_{i}
$$

and $r$ is reduced w.r.t. all the $f_{i}$.

The definition below is related to this theorem.

\section{Definition 2.14}

If all $r$ which satisfies the conditions of Theorem 2.3 are equal then $p$ reduces to $r$ modulo $F$ which we write $p \longrightarrow_{F} r$.

The algorithm for calculating Gröbner bases uses the so called S-polynomials.

\section{Definition 2.15}

Let $f_{1}, f_{2} \in k\left[x_{1}, \ldots, x_{n}\right]$. Then the $S$-polynomial of $f_{1}$ and $f_{2}$ is

$$
S\left(f_{1}, f_{2}\right)=h_{1} f_{1}-h_{2} f_{2}
$$

where

$$
h_{1}=L C f_{2} \frac{\operatorname{lcm}\left(L M f_{1}, L M f_{2}\right)}{L M f_{1}}, \quad h_{2}=L C f_{1} \frac{\operatorname{lcm}\left(L M f_{1}, L M f_{2}\right)}{L M f_{2}}
$$

and $\operatorname{lcm}(\cdot, \cdot)$ denotes the least common monomial.

The Gröbner base algorithm can now be given as follows given some set of polynomials $F$. 


\section{Algorithm 2.1}

1. Make $F$ auto-reduced.

2. Add to $F$ the $S$-polynomial of two elements in $F$.

3. If all $S$-polynomials reduce to zero modulo $F$ then $F$ is a Gröbner base. Otherwise return to step 1.

The following theorem ensures that the result of this algorithm is a Gröbner base.

\section{Theorem 2.4}

The set $G=\left\{g_{1}, \ldots, g_{m}\right\}$ is a Gröbner base for $\langle G\rangle$ iff

$$
S\left(g_{i}, g_{j}\right) \longrightarrow_{G} 0
$$

A property of Gröbner bases that we have mentioned earlier is that we can eliminate variables from a set of polynomial equations. How this elimination is performed is given in the following theorem.

\section{Theorem 2.5}

Let $\mathcal{I}$ be an ideal in $k\left[x_{1}, \ldots, x_{n}\right]$ and partition $x_{1}, \ldots, x_{n}$ into two disjoint sets $A$ and $B$. Then if $G$ is a Gröbner base for $\mathcal{I}$ with a lexicographic term ordering where $A<B$ then $k[A] \cap G$ is a Gröbner base for $k[A] \cap \mathcal{I}$ w.r.t. the term ordering given by $<$ on $k[A]$.

What this theorem says is that with a proper choice of term ordering we can find out if there are elements in $\mathcal{I}$ which are polynomial in the variables $A$ only. If this is the case we also get a generating set for the corresponding ideal. We give a simple example.

\section{Example 2.1}

Consider the following set if polynomial equations

$$
\begin{aligned}
& x^{2}+y+z=1 \\
& x+y^{2}+z=1 \\
& x+y+z^{2}=1
\end{aligned}
$$

Calculating a Gröbner base for the polynomials

$$
\left\{x^{2}+y+z-1, x+y^{2}+z-1, x+y+z^{2}-1\right\}
$$

with the term ordering $x>y>z$ gives the result

$$
\left\{x+y+z^{2}-1, y^{2}+z-y-z^{2}, 2 y z^{2}+z^{4}-z^{2}, z^{6}-4 z^{4}+4 z^{3}-z^{2}\right\}
$$

From this it is clear that

$$
z^{6}-4 z^{4}+4 z^{3}-z^{2} \in\left\langle x^{2}+y+z-1, x+y^{2}+z-1, x+y+z^{2}-1\right\rangle
$$

and we have eliminated $x$ and $y$ from the set of equations (13).

This example indicates that Gröbner bases can be interpreted as a generalization of Gaussian elimination. The algorithm for calculating Gröbner bases has been implemented in every major computer algebra language such as MAPLE and Mathematica. 


\subsection{Differential algebra}

The theory presented in this section is gathered from [4] and the proofs can be found either there or in the books $[9,8]$.

The main difference between differential and commutative algebra is that we add differentiation w.r.t. time, $\partial / \partial t$, to the set of allowed operations.

\section{Definition 2.16}

$A$ differential field $k$ is a field satisfying the conditions

$$
\begin{aligned}
\forall a \in k, \quad \frac{\partial a}{\partial t} & =\dot{a} \in k \\
\forall a, b \in k, \quad \frac{\partial}{\partial t}(a+b) & =\dot{a}+\dot{b} \\
\forall a, b \in k, \quad \frac{\partial}{\partial t}(a b) & =\dot{a} b+a \dot{b}
\end{aligned}
$$

Here and in the sequel will the "dot" notation be used instead of $\partial / \partial t$ to denote differentiation with respect to time. We also make a few more definitions regarding differential fields which are the differential variants of some of the commutative definitions in the previous section.

\section{Definition 2.17}

If the differential field $k$ is a subset of the differential field $K$ then $K$ is said to be a differential extension field of $k$. This is denoted by $K / k$. If $K$ is a differential extension field of $k$ and $\alpha \subset K$ then the smallest differential field containing $k \cup \alpha$ is called the differential field obtained by adjoining $\alpha$ to $k$ and is denoted $k\langle\alpha\rangle$.

We now consider a definition of dynamics based on differential algebra.

\section{Definition 2.18}

A dynamic system is a finitely generated differential algebraic extension $D / k\langle u\rangle$ where $u=\left\{u_{1}, \ldots, u_{m}\right\}$ can be viewed as inputs and the outputs $y$ is chosen as some finite set $y=\left\{y_{1}, \ldots, y_{p}\right\}$ in $D$.

Using this definition a more general form of state space description than (22) can be made.

\section{Theorem 2.6}

Let $K / k\langle u\rangle$ be a dynamic system according to definition 2.18. Then there exists a generalized state $x=\left\{x_{1}, \ldots, x_{n}\right\}$ for some finite integer $n$ and polynomials $A_{j}(\cdot)$ and $B_{i}(\cdot)$ such that

$$
\begin{aligned}
A_{j}\left(\dot{x}_{j}, x, u, \dot{u}, \ldots, u^{\left(\alpha_{j}\right)}\right) & =0, \quad j=1, \ldots, n \\
B_{i}\left(y_{i}, x, u, \dot{u}, \ldots, u^{\left(\beta_{i}\right)}\right) & =0, \quad i=1, \ldots, p
\end{aligned}
$$

A theorem which is important when considering differentially algebraic extensions is given below.

Theorem 2.7

Let $L / K$ be a finitely generated differentially generated algebraic extension such that $K$ contains non constant elements. Then there exists an element $\xi \in L$ such that $L=K\langle\xi\rangle$. The element $\xi$ is called a differentially primitive element. 


\section{A Generalized Normal Form}

Here we will study how the normal form of affine state space descriptions can generalized to the more general dynamics of Definition 2.18.

\subsection{The normal form of affine state space systems}

The theory in this section is collected from [7]. Consider the SISO nonlinear system which is described by the following state equations

$$
\begin{aligned}
& \dot{x}=f(x)+g(x) u \\
& y=h(x)
\end{aligned}
$$

where $x \in \mathbb{R}^{n}$. The system (22) is said to have relative degree $r \leq n$ if

$$
\begin{aligned}
\text { (i) } L_{g} L_{f}^{k} h(x) & \equiv 0, \quad k=0, \ldots, r-2 \\
\text { (ii) } L_{g} L_{f}^{r-1} h(x) & \not \equiv 0
\end{aligned}
$$

at some point $x^{0}$. Here $L_{f}$ and $L_{g}$ denote the Lie derivatives in the directions $f(x)$ and $g(x)$ respectively. Using the notation

$$
d h(x)=\left(\frac{\partial h(x)}{\partial x_{1}}, \frac{\partial h(x)}{\partial x_{2}}, \ldots, \frac{\partial h(x)}{\partial x_{n}}\right)
$$

the following lemma can be shown to hold.

Lemma 3.1 The row vectors

$$
d h\left(x^{0}\right), d L_{f} h\left(x^{0}\right), \ldots, d L_{f}^{r-1} h\left(x^{0}\right)
$$

are linearly independent.

We can now perform the change of variables locally around $x^{0}$ where

$$
\begin{aligned}
\phi_{1}(x) & =h(x) \\
\phi_{2}(x) & =L_{f} h(x) \\
& \vdots \\
\phi_{r}(x) & =L_{f}^{r-1} h(x)
\end{aligned}
$$

If $r<n$ then $\phi_{i}(x), i=r+1, \ldots, n$ can be found so that the change of variables becomes invertible. In particular these functions can be chosen so that

$$
L_{g} \phi_{i}(x)=0, \quad i=r+1, \ldots, n
$$

We see that finding $\phi_{i}(x), i=r+1, \ldots, n$ such that (27) is satisfied involves solving $n-r$ partial differential equations which is of course difficult in general. However, if we do not bother with the condition (27) the invertible transformation is most often very easy to find. With this transformation the new state 
equations becomes

$$
\begin{aligned}
\dot{z}_{1} & =z_{2} \\
\dot{z}_{2} & =z_{3} \\
& \vdots \\
\dot{z}_{r-1} & =z_{r} \\
\dot{z}_{r} & =L_{f}^{r} h\left(\Phi^{-1}(z)\right)+L_{g} L_{f}^{r-1} h\left(\Phi^{-1}(z)\right) u \\
\dot{z}_{r+1} & =q_{r+1}(z)+p_{r+1}(z) u \\
& \vdots \\
\dot{z}_{n} & =q_{n}(z)+p_{n}(z) u \\
y & =z_{1}
\end{aligned}
$$

This is known as the normal form of the system. We note that with the condition (27) we get $p_{i}(x) \equiv 0, i=r+1, \ldots, n$.

\subsection{A generalization of the normal form}

In this section we repeat the results of [6] where all proofs that are left out and further constructive details can be found. We consider a dynamics $D / k\langle u\rangle$ which has differential transcendence degree 1 and trdeg $D / k\langle u\rangle=n$. In generalized state space form we thus get

$$
\begin{aligned}
A_{1}\left(\dot{x}_{1}, x, u, \dot{u}, \ldots, u^{\left(\alpha_{1}\right)}\right) & =0 \\
& \vdots \\
A_{n}\left(\dot{x}_{n}, x, u, \dot{u}, \ldots, u^{\left(\alpha_{n}\right)}\right) & =0 \\
B\left(y, x, u, \dot{u}, \ldots, u^{(\beta)}\right) & =0
\end{aligned}
$$

where we we for simplicity say that the system has only one output. Since we want to be able to have a unique description of the output we assume that the condition

$$
\frac{\partial B(y, x)}{\partial y} \neq 0
$$

is fulfilled. Our goal is then to try to find a generalization of the normal form (28). The first thing we must do is then to generalize the Lie derivative and the relative degree. The first thing we note is that if the output polynomial explicitly depends on $u$ and its derivatives we have a case which do not correspond to the affine case. The natural thing is then to say that such a system has relative degree zero and that (29) is already in normal form. If this is not the case we must study the following polynomial

$$
\frac{d B(y, x)}{d t}=\sum_{i=1}^{n} \frac{\partial B(y, x)}{\partial x_{i}} \dot{x}_{i}+\frac{\partial B(y, x)}{\partial y} \dot{y}
$$

The correspondence to the Lie derivative (23) would then be if all $\dot{x}_{i}$ and the $y$ could be eliminated from (31). Obviously this can be done with Gröbner bases as given in the definition below. 


\section{Definition 3.1}

Suppose that we have a system (29) where the output polynomial does not depend explicitly on $u$ and that $G_{1}$ is a Gröbner base for the polynomials

$$
\left\{A_{1}(\cdot), \ldots, A_{n}(\cdot), B(y, x), d B(y, x) / d t\right\}
$$

under the term ordering

$$
x_{n}<\cdots<x_{1}<\dot{y}<u<\dot{u}<\cdots<u^{\left(\alpha_{\max }\right)}<y<\dot{x}_{n}<\cdots<\dot{x}_{1}
$$

where $\alpha_{\max }=\max \left\{\alpha_{1}, \ldots, \alpha_{n}\right\}$. The generalized Lie derivative of $y$ w.r.t.

$$
A=\left\{A_{1}(\cdot), \ldots, A_{n}(\cdot)\right\}
$$

is then defined as

$$
L_{A} B(y, x)=G_{1} \cap k\left[x_{1}, \ldots, x_{n}, u, \dot{u}, \ldots, u^{\left(\alpha_{\max }\right)}, \dot{y}\right]
$$

with

$$
\frac{\partial L_{A} B(y, x)}{\partial \dot{y}} \neq 0
$$

The iterated generalized Lie derivative $L_{A}^{i} B(y, x)$ with $i>1$ is defined as

$$
L_{A}^{i} B(y, x)=G_{i} \cap k\left[x_{1}, \ldots, x_{n}, u, \dot{u}, \ldots, u^{\left(\alpha_{\max }\right)}, y^{(i)}\right]
$$

with

$$
\frac{\partial L_{A}^{i} B(y, x)}{\partial y^{(i)}} \neq 0
$$

Here $G_{i}$ is the Gröbner base of

$$
\left\{A_{1}(\cdot), \ldots, A_{n}(\cdot), B(y, x), L_{A} B(y, x), \ldots, L_{A}^{i-1} B(y, x), d L_{A}^{i-1} B(y, x) / d t\right\}
$$

w.r.t. the term ordering

$$
x_{n}<\cdots<x_{1}<y^{(i)}<u<\dot{u}<\cdots<u^{\left(\alpha_{\max }\right)}<\cdots<\dot{y}<y<\dot{x}_{n}<\cdots<\dot{x}_{1}
$$

Here and in the sequel will $k$ denote the field of real numbers. With this definition we can define a relative degree for systems of the form (29).

\section{Definition 3.2}

A system (29) has generalized relative degree $r \geq 1$ if $L_{A}^{i} B(y, x), i=1, \ldots, r$ consist of only one polynomial for each $i$ and it holds that

$$
\frac{\partial L_{A}^{i} B(y, x)}{\partial u^{(j)}} \equiv 0, \forall j \geq 0, i=1, \ldots, r-1
$$

and

$$
\frac{\partial L_{A}^{r} B(y, x)}{\partial u^{(j)}} \neq 0, \text { for some } j \geq 0
$$

In order to justify the names generalized Lie derivative and generalized relative degree we must now show that the usual definitions of these concepts follow as special cases. 


\section{Theorem 3.1}

Suppose that we have a system (22) with $f(x), g(x)$ and $h(x)$ polynomials in the ring $k\left[x_{1}, \ldots, x_{n}\right]$. Then this system has relative degree $r$ iff it has generalized relative degree $r$. The Lie derivatives $L_{f}^{i} h(x), i=0, \ldots, r-1$ are given as the solutions to

$$
L_{A}^{i}(y-h(x))=0, \quad i=0, \ldots, r-1
$$

w.r.t. $y^{(i)}, i=1, \ldots, r-1$. Furthermore, $L_{f}^{r} h(x)+L_{g} L_{f}^{r-1} h(x) u$ is the solution to

$$
L_{A}^{r}(y-h(x))=0
$$

w.r.t. $y^{(r)}$.

The question is now how these Lie derivatives can be used for finding a normal form for our system (29). Given a relative degree $r \geq 1$ we have a set of polynomials

$$
\left\{p_{1}(y, x), p_{2}(\dot{y}, x), \ldots, p_{r}\left(y^{(r-1)}, x\right)\right\}
$$

and in correspondence with the normal form (28) we want to perform a transformation such that

$$
z_{1}=y, z_{2}=\dot{y}, \ldots, z_{r}=y^{(r-1)}
$$

The $y^{(i)}, i=0, \ldots, r-1$ can now be shown to have the following property.

\section{Theorem 3.2}

Suppose that the dynamic system (29) has generalized relative degree $r \geq 1$. Then $r \leq n$ and the output derivatives $y^{(i)}, i=0, \ldots, r-1$ are algebraically independent over $k\langle u\rangle$.

Remark 3.1 This theorem has a nice interpretation in the affine polynomial case namely that the proof of Lemma 3.1 follows directly from Theorem 3.2 and Theorem 2.2.

Since trdeg $D / k\langle u\rangle=n$ we know that there exists $z_{i} \in D / k\langle u\rangle, i=r+$ $1, \ldots, n$ such that with $z_{i}=y^{(i-1)}, i=1, \ldots, r$ the set $\left\{z_{1}, \ldots, z_{n}\right\}$ becomes a transcendence basis for $D / k\langle u\rangle$. The question is how the $z_{r+1}, \ldots, z_{n}$ can be chosen. The lemma below gives a partial answer to this question.

Lemma 3.2 Suppose that the dynamic system (29) has generalized relative degree $r \leq n$. Then there exists $n-r$ different integers $i_{r+1}, \ldots, i_{n}$ between 1 and $n$ such that with

$$
z_{j}= \begin{cases}y^{(j-1)}, & j=1, \ldots, r \\ x_{i_{j}}, & j=r+1, \ldots, n\end{cases}
$$

the set $\left\{z_{1}, \ldots, z_{n}\right\}$ becomes a transcendence basis for $D / k\langle u\rangle$.

A constructive note that must be made now is that in Lemma 3.2 it is not shown how to choose the $x_{i}$ which are algebraically independent over the field $k\langle u\rangle\left(z_{1}, \ldots, z_{j}\right), j=r, \ldots, \bar{r}-1$. These $x_{i}$ can be found with Gröbner bases in a way which is described in [6]. The main tool for performing the transformation 
the system (29) will of course also be Gröbner bases and the generalized normal form becomes

$$
\begin{aligned}
\dot{z}_{1} & =z_{2} \\
\dot{z}_{2} & =z_{3} \\
& \vdots \\
\dot{z}_{r-1} & =z_{r} \\
q_{r}\left(\dot{z}_{r}, z, u, \dot{u}, \ldots, u^{\left(\nu_{r}\right)}\right) & =0 \\
q_{r+1}\left(\dot{z}_{r+1}, z, u, \dot{u}, \ldots, u^{\left(\nu_{r+1}\right)}\right) & =0 \\
& \vdots \\
q_{n}\left(\dot{z}_{n}, z, u, \dot{u}, \ldots, u^{\left(\nu_{n}\right)}\right) & =0 \\
y & =z_{1}
\end{aligned}
$$

Due to the fact that if we would have performed the calculations above for an affine system (22) we would get (28) we can call this a generalized normal form for $(29)$.

\section{Sliding Mode Control for Generalized State Space Descriptions}

In [12] a method for designing sliding mode controllers for generalized state space descriptions (29) is given. However, this method has several drawbacks. One is that one is restricted to zeroing the differentially primitive element and another is that one must solve a high order differential equation to find the sliding control $u$. We will now study if the generalized normal form defined in the previous section can be used for reducing these problems.

\subsection{Sliding Mode Control using the Differentially Primi- tive Element}

Here we will repeat the results of [12]. First of all it is shown in [2] how Theorem 2.7 can be used for getting a more suitable form for the dynamics.

\section{Theorem 4.1}

Suppose that $\xi$ is a primitive element of the dynamics $D / k\langle u\rangle$. Then $x_{1}=$ $\xi, \ldots, x_{n}=\xi^{(n)}$ qualifies a generalized state vector and the corresponding state space description becomes

$$
\begin{aligned}
\dot{x}_{1} & =x_{2} \\
\dot{x}_{2} & =x_{3} \\
& \vdots \\
\dot{x}_{n-1} & =x_{n} \\
C\left(\dot{x}_{n}, x_{1}, \ldots, x_{n}, u, \dot{u}, \ldots, u^{(\nu)}\right) & =0
\end{aligned}
$$

This is known as the generalized controller canonical form.

What we want to achieve is to keep the primitive element $\xi$ zero. As before we introduce an auxiliary output defined as

$$
\sigma=c_{1} x_{1}+\cdots+c_{n-1} x_{n-1}+x_{n}
$$


with the $c_{i}$ chosen so that

$$
c_{1}+c_{2} s+\cdots+c_{n-1} s^{n-2}+s^{n-1}
$$

is Hurwitz. This output will be our ideal sliding dynamics. We now impose the following discontinuous dynamics for our sliding manifold

$$
\dot{\sigma}=-W \operatorname{sign}(\sigma)
$$

where $W>0$ and

$$
\operatorname{sign}(\sigma)=\left\{\begin{array}{rr}
1, & \sigma>0 \\
-1, & \sigma<0
\end{array}\right.
$$

From [11] we now know that we then achieve a sliding regime in finite time $T=W^{-1}|\sigma(0)|$. Using (50) and (49) we see that this is achieved by choosing an input that satisfies the differential equation

$$
C\left(\dot{x}_{n}, x_{1}, \ldots, x_{n}, u, \dot{u}, \ldots, u^{(\nu)}\right)=0
$$

with $\dot{x}_{n}$ replaced by

$$
-c_{1} x_{2}-c_{2} x_{3}-\cdots-c_{n-1} x_{n}-W \operatorname{sign}\left(c_{1} x_{1}+\cdots+c_{n-1} x_{n-1}+x_{n}\right)
$$

Then when $\sigma=0$ has been reached we get from (50) that $x_{n}$ no longer qualifies as a state variable which gives the remaining dynamics

$$
\begin{aligned}
\dot{x}_{1} & =x_{2} \\
\dot{x}_{2} & =x_{3} \\
& \vdots \\
\dot{x}_{n-1} & =x_{n}=-c_{1} x_{1}-\cdots-c_{n-1} x_{n-1}
\end{aligned}
$$

Since (51) is Hurwitz we immediately get that we get an asymptotically stable motion towards $x_{1}=\cdots=x_{n}=0$ which was our original goal. From (54) and (55) it is also clear that the equivalent control on $\sigma=0$ will tend towards the differential equation

$$
C\left(0,0, \ldots, 0, u_{E Q}, \dot{u}_{E Q}, \ldots, u_{E Q}^{(\nu)}\right)=0
$$

We can note that in [3] (57) is defined as the zero dynamics associated with zeroing the primitive element.

\subsection{Sliding Mode Control using the Generalized Normal Form}

The basic ideas of designing a sliding mode controller by utilizing the generalized normal form will essentially be the same as the ones used for the generalized controller canonical form. Again consider a system (29). Our objective is to keep $y=0$ so the sliding manifold that we wish to achieve is $B(0, x)=0$. Accordingly we set the sliding surface coordinate to be $\sigma=B(0, x)$ and as before we say that this coordinate should satisfy (52). Now suppose that if we treat $\sigma$ as an 
auxiliary output our system gets relative degree 1, i.e., $L_{A}(\sigma-B(0, x))$ depends explicitly on the input $u$. We set

$$
L_{A}(\sigma-B(0, x))=p\left(\dot{\sigma}, x, u, \dot{u}, \ldots, u^{\left(\nu_{\sigma}\right)}\right)
$$

As above we can then conclude that a sliding regime is achieved by choosing an input which is a solution to the differential equation

$$
p\left(-W \operatorname{sign}(B(0, x)), x, u, \dot{u}, \ldots, u^{\left(\nu_{\sigma}\right)}\right)=0
$$

if this equation can be solved w.r.t. $u$.

The case when we with our $\sigma=B(0, x)$ get $r>1$ is handled as follows. We define an auxiliary output as

$$
\psi=c_{1} \sigma+\cdots+c_{r-1} \sigma^{(r-2)}+\sigma^{(r-1)}
$$

with the $c_{i}$ chosen so that

$$
c_{1}+c_{2} s+\cdots+c_{r-1} s^{r-2}+s^{r-1}
$$

is Hurwitz. Using $\sigma-B(0, x), \ldots, L_{A}^{r-1}(\sigma-B(0, x))$ we can give the output function in terms of $x$ instead as

$$
B_{\psi}(\psi, x)=0
$$

Since we wish to keep $\psi=0$ we introduce a sliding coordinate $\eta$ as

$$
\eta=B_{\psi}(0, x)
$$

It is obvious that with an output defined as (63) we get relative degree 1 . We then get that

$$
L_{A}(\eta-B(0, x))=q\left(\dot{\eta}, x, u, \dot{u}, \ldots, u^{\left(\nu_{\eta}\right)}\right)
$$

Imposing the dynamics

$$
\dot{\eta}=-W \operatorname{sign}(\eta)
$$

gives that the input that achieves a sliding regime on $\eta=0$ is given by

$$
q\left(-W \operatorname{sign}\left(B_{\psi}(0, x)\right), x, u, \dot{u}, \ldots, u^{\left(\nu_{\eta}\right)}\right)=0
$$

which in turn will give $\psi=0$. Now consider the generalized normal form (48) which we would get with the output $\sigma=B(0, x)$. When we then reach $\psi=0$ it is clear from (60) that in the new variables we get that

$$
c_{1} z_{1}+\cdots+c_{r-1} z_{r-1}+z_{r}=0
$$

so that one state variable, say $z_{r}$, do no longer qualify as a state space variable. The normal form (48) then becomes

$$
\begin{aligned}
\dot{z}_{1} & =z_{2} \\
\dot{z}_{2} & =z_{3} \\
& \vdots \\
\dot{z}_{r-1} & =z_{r}=-c_{1} z_{1}-\cdots-c_{r-1} z_{r-1} \\
q_{r+1}\left(\dot{z}_{r+1}, z, u, \dot{u}, \ldots, u^{\left(\nu_{r+1}\right)}\right) & =0 \\
& \vdots \\
q_{n}\left(\dot{z}_{n}, z, u, \dot{u}, \ldots, u^{\left(\nu_{n}\right)}\right) & =0 \\
\sigma & =z_{1}
\end{aligned}
$$


with the $u$ given by (66). Due to our choice of $c_{i}$ we get an asymptotically stable motion towards

$$
\sigma=z_{1}=z_{2}=\cdots=z_{r}=0
$$

which means that we approach $B(0, x)=0$. Under the condition that (36) and (38) holds for $i=0$ we thus achieve $y=0$ asymptotically.

What about the internal dynamics given by the last $n-r$ equations in (68)? This dynamics will together with the equation defining the equivalent control asymptotically approach

$$
\begin{aligned}
q_{r}\left(0, \bar{z}, u_{E Q}, \dot{u}_{E Q}, \ldots, u_{E Q}^{\left(\nu_{r}\right)}\right) & =0 \\
q_{r+1}\left(\dot{z}_{r+1}, \bar{z}, u_{E Q}, \dot{u}_{E Q}, \ldots, u_{E Q}^{\left(\nu_{r+1}\right)}\right) & =0 \\
q_{n}\left(\dot{z}_{n}, \bar{z}, u_{E Q}, \dot{u}_{E Q}, \ldots, u_{E Q}^{\left(\nu_{n}\right)}\right) & =0
\end{aligned}
$$

where $\bar{z}=\left(0, \ldots, 0, z_{r+1}, \ldots, z_{n}\right)$. In accordance with (57) we define (70) to be the zero dynamics associated with zeroing $y$.

Comparing with the construction of a sliding control law using the differentially primitive element as described in the previous section we have obviously improved two things. First of all we can now achieve sliding regimes on manifolds more general than manifolds given by a differentially primitive element. We can even assume that under some conditions the output which we wish to keep zero can be given implicitly. Also a comparison with the differential equation giving the control laws it is seen that comparing (54) with (59) or (66) we see that the latter equations are of a degree in $u$ that is never higher than that of (54). In fact for systems with a low relative degree the degree of (59) or (66) may be much lower than (54). This is an advantage when the practical computations are to be performed. A problem may be that comparing (57) and (70) when $r<n$ one gets a more complicated zero dynamics to study with the generalized normal form.

It is important to note here that there may of course be situations when a control cannot be calculated from the differential equations (59) or (66). These equations are thus to be interpreted as if a real solution exist then does the control give a sliding regime on the corresponding manifold. Otherwise one may try with another manifold. This problem is of course the same for the sliding mode control law given by (54), (55).

\section{Conclusions}

In the report it has been shown how the generalized normal form can be used in the context of sliding mode control. As a comparison with the result of [12] we show how to construct sliding controllers for manifold which are more general than the differentially primitive element of the dynamics. Also the differential equation for the control law is shown never to have a higher degree than the one we get using the differentially primitive element but it may very well be significantly lower. This can obviously simplify the practical computations. 


\section{Acknowledgment}

This work was financially supported by the Swedish Research Council for Engineering Sciences (TFR).

\section{References}

[1] D. Cox, J. Little, and D. O'Shea. Ideals, Varieties, and Algorithms An Introduction to Computational Algebraic Geometry and Commutative algebra. Undergraduate texts in mathematics. Springer, 1992.

[2] M. Fliess. Generalized controller canonical forms for linear and nonlinear dynamics. Trans. Aut. Control, AC-35(9):994-1001, 1990.

[3] M. Fliess. What the Kalman state variable representation is good for. In Proc. 29th IEEE Conf. on Decision and Control, pages 1282-1287, Honolulu, Hawaii, 1990.

[4] M. Fliess and S. T. Glad. An algebraic approach to linear and nonlinear control. In H. L. Trentelman and J. C. Willems, editors, Essays on Control: Perspectives in the Theory and its Applications, pages 223-267. Birkhäuser, Boston, 1993.

[5] K. Forsman. Constructive Commutative Algebra in Nonlinear Control Theory. PhD thesis, Dept. of Electrical Engineering, Linköping University, S-581 83 Linköping, Sweden, 1991.

[6] H. Fortell. A normal form for generalized state space descriptions. Technical Report LiTH-ISY-R-1738, Dept. of Electrical Engineering, Linköping University, S-581 83 Linköping, Sweden, 1995. Available by anonymous ftp 130.236.24.1.

[7] A. Isidori. Nonlinear Control Systems. Springer-Verlag, second edition, 1989.

[8] E. R. Kolchin. Differential Algebra and Algebraic Groups. Academic Press, 1973.

[9] J. F. Ritt. Differential Algebra. Dover Publications, New York, 1950.

[10] H. Sira-Ramirez. Structure at infinity, zero dynamics and normal forms of systems undergoing sliding motions. Int. J. Systems Sci., 21(4):665-674, 1990.

[11] H. Sira-Ramirez. Dynamical discontinuous feedback control of non-linear systems. In Proc. NOLCOS '92, pages 471-476, Bordeaux, France, 1992.

[12] H. Sira-Ramirez. On the dynamical sliding mode control of nonlinear systems. Int. J. Control, 57(5):1039-1061, 1993. 